\title{
Characterizing Waiting Room Time, Treatment Time, and Boarding Time in the Emergency Department Using Quantile Regression
}

\author{
Ru Ding, MS, Melissa L. McCarthy, ScD, Jeffrey S. Desmond, MD, Jennifer S. Lee, MD, Dominik
} Aronsky, MD, PhD, and Scott L. Zeger PhD

\begin{abstract}
Objectives: The objective was to characterize service completion times by patient, clinical, temporal, and crowding factors for different phases of emergency care using quantile regression (QR).

Methods: A retrospective cohort study was conducted on 1-year visit data from four academic emergency departments (EDs; $N=48,896-58,316$ ). From each ED's clinical information system, the authors extracted electronic service information (date and time of registration; bed placement, initial contact with physician, disposition decision, ED discharge, and disposition status; inpatient medicine bed occupancy rate); patient demographics (age, sex, insurance status, and mode of arrival); and clinical characteristics (acuity level and chief complaint) and then used the service information to calculate patients' waiting room time, treatment time, and boarding time, as well as the ED occupancy rate. The 10th, 50th, and 90th percentiles of each phase of care were estimated as a function of patient, clinical, temporal, and crowding factors using multivariate QR. Accuracy of models was assessed by comparing observed and predicted service completion times and the proportion of observations that fell below the predicted 10th, 50th, and 90th percentiles.
\end{abstract}

Results: At the 90th percentile, patients experienced long waiting room times (105-222 minutes), treatment times (393-616 minutes), and boarding times (381-1,228 minutes) across the EDs. We observed a strong interaction effect between acuity level and temporal factors (i.e., time of day and day of week) on waiting room time at all four sites. Acuity level 3 patients waited the longest across the four sites, and their waiting room times were most influenced by temporal factors compared to other acuity level patients. Acuity level and chief complaint were important predictors of all phases of care, and there was a significant interaction effect between acuity and chief complaint. Patients with a psychiatric problem experienced the longest treatment times, regardless of acuity level. Patients who presented with an injury did not wait as long for an ED or inpatient bed. Temporal factors were strong predictors of service completion time, particularly waiting room time. Mode of arrival was the only patient characteristic that substantially affected waiting room time and treatment time. Patients who arrived by ambulance had shorter wait times but longer treatment times compared to those who did not arrive by ambulance. There was close agreement between observed and predicted service completion times at the 10th, 50th, and 90th percentile distributions across the four EDs.

Conclusions: Service completion times varied significantly across the four academic EDs. QR proved to be a useful method for estimating the service completion experience of not only typical ED patients, but also the experience of those who waited much shorter or longer. Building accurate models of ED service completion times is a critical first step needed to identify barriers to patient flow, begin the process of reengineering the system to reduce variability, and improve the timeliness of care provided.

ACADEMIC EMERGENCY MEDICINE 2010; 17:813-823 @ 2010 by the Society for Academic Emergency Medicine

Keywords: emergency department, length of stay, quantile regression, prediction

From the Department of Emergency Medicine, Johns Hopkins University School of Medicine (RD, MLM), Baltimore, MD; the Department of Emergency Medicine, University of Michigan School of Medicine (JSD), Ann Arbor, MI; the Department of Emergency Medicine, George Washington University School of Medicine (JSL), Washington, DC; the Department of Biomedical Informatics and the Department of Emergency Medicine, Vanderbilt University Medical Center (DA), Nashville, TN; and the Department of Biostatistics, Johns Hopkins University Bloomberg School of Public Health (SLZ), Baltimore, MD.

Received November 5, 2009; revisions received January 21 and February 17, 2010; accepted February 19, 2010.

This study was funded in part by grant, 1K01HS017957-01, which Dr. McCarthy received from the Agency for Healthcare Research and Quality.

Supervising Editor: James E. Olson, PhD.

Address for correspondence and reprints: Melissa L. McCarthy; e-mail: mmccarth@jhmi.edu. 
$\mathrm{S}$ ervice completion times are a hallmark of service industries such as telecommunication, transportation, and food and beverage sectors. In the emergency department (ED), waiting room time, treatment time, and boarding time are major phases of emergency care. Many EDs consider these service completion times measures of ED and hospital efficiency and monitor them to identify delays in care. Few investigators have developed models that characterize the completion times of different phases of emergency care. Asaro et al. ${ }^{1}$ used multivariate linear regression techniques to estimate ED waiting room time, treatment time, and boarding time for patients who were admitted or discharged from the main ED or urgent care area. Similarly, McCarthy et al. ${ }^{2}$ applied discrete-time survival analysis to evaluate the effect of crowding on the different phases of ED care. Both studies estimated the influence of various patient, temporal, and system factors on the mean or median completion times for different phases of emergency care. However, the distribution of ED length of stay (LOS) is highly positively skewed; that is, the deviations are much greater above the median than below. Focusing only on what happens on average, or in the middle, may provide an incomplete picture of the substantial variation that can exist among ED patients. To fully understand the LOS experience for all patients requires an analysis of the middle as well as both tails of the distribution.

The purpose of this study was to characterize service completion times at four academic EDs using quantile regression $(\mathrm{QR})$. QR offers a richer inference than other approaches because the LOS experience can be described at any percentile of the distribution. In this study, we used QR to develop models that accurately predict the 10th, 50th, and 90th percentiles of ED waiting room time, treatment time, and boarding time. We chose these particular percentiles of the distribution because in addition to characterizing a typical ED LOS experience (i.e., 50th percentile), we wanted to understand the experience of patients treated among the fast- est (10th percentile) and among the slowest (90th percentile). We based the QR models on patient demographic, clinical, temporal, and crowding factors available at triage so that in the future, the model estimates could be provided in real time to patients at the end of the triage process so that they would have a realistic expectation of how long it would take to complete different phases of their emergency care.

\section{METHODS}

\section{Study Design}

This study relied on a retrospective cohort design that included all visits to each of four EDs during a 1-year period. We extracted service time data as well as patient and clinical information that were collected during triage or registration from the clinical information systems of each ED. We used QR to estimate the 10th, 50th, and 90th percentiles for each phase of care. Because a detailed description of the study setting, selection of participants, and data collection procedures is available elsewhere, ${ }^{2}$ only a brief summary of these topics follows. The study was reviewed and approved by the institutional review board at each site.

\section{Study Setting and Population}

The four study EDs are each part of tertiary care, academic medical facilities with Level I trauma centers. The annual ED volumes at each site range from 50,000 to 62,000 (see Table 1). However, the EDs differ substantially in other ways such as ED bed capacity (26 to 41 beds in main ED); inpatient medicine bed capacity, which includes all beds assigned to the divisions within the department of medicine at each academic site (range of 224 to 461 beds); existence of an observation unit (two of the four EDs); separate pediatric ED (three of the four EDs); dedicated laboratory (one of the four EDs); and whether boarders, defined as admitted ED patients waiting for a hospital bed, are managed by emergency physicians (EPs; two of the four EDs). There

\begin{tabular}{|c|c|c|c|c|}
\hline Facility Characteristics & Site A & Site B & Site C & Site D \\
\hline Annual ED volume & 57,691 & 56,832 & 50,824 & 61,187 \\
\hline \multicolumn{5}{|l|}{ Bed capacity } \\
\hline Main ED & 32 & 27 & 41 & 26 \\
\hline Fast track & 7 & 5 & 4 & 10 \\
\hline Observation & 14 & 16 & 0 & 0 \\
\hline Inpatient medicine & 224 & 287 & 461 & 246 \\
\hline \multicolumn{5}{|l|}{ Median staffing (IQR) } \\
\hline Doctors per hour & $3(1-4)$ & $3(2-3)$ & $3(2-4)$ & $2(1-2)$ \\
\hline Residents per hour & $4(4-5)$ & $3(2-3)$ & $4(4-5)$ & $4(4-4)$ \\
\hline Nurses per hour & $15(14-17)$ & $21(18-22)$ & $14(12-15)$ & $10(8-11)$ \\
\hline Hourly staffing/bed & $0.45(0.42-0.47)$ & $0.54(0.49-0.60)$ & $0.51(0.45-0.53)$ & $0.50(0.47-0.53)$ \\
\hline Hourly staffing/patient & $0.52(0.45-0.62)$ & $0.56(0.47-0.68)$ & $0.58(0.48-0.74)$ & $0.43(0.35-0.54)$ \\
\hline EPs care for ED boarders & Yes & No & No & Yes \\
\hline \multicolumn{5}{|c|}{$\begin{array}{l}\text { Median hourly occupancy rate, } \\
\%(I Q R)\end{array}$} \\
\hline ED & $86(70-102)$ & $98(78-121)$ & 86 (65-105) & $116(89-143)$ \\
\hline Inpatient medicine beds & $83(77-87)$ & $90(86-93)$ & $78(75-80)$ & $83(77-87)$ \\
\hline
\end{tabular}


was also significant variation among the sites in terms of staffing, particularly nurse staffing (median range 10-21). The median hourly ED occupancy rate (range $86 \%-116 \%$ ) and inpatient medicine bed occupancy rate (range 78\%-90\%) also demonstrated considerable variability across the four sites.

The patient populations of the study EDs differ substantially on a few key characteristics. Site A treats a higher proportion of uninsured $(37 \%)$ compared to the other three sites (range 5\%-21\%). Site D treats a higher percentage (45\%) of low-acuity patients (i.e., Levels 4 and 5 according to the Emergency Severity Index [ESI]) compared to the other sites (range 14\%$25 \%$ ). The hospital admission rate varies substantially by site (range 19\%-30\%). Other patient characteristics are fairly similar across the sites, including age (range $21 \%-29 \%>55$ years), female sex (range $52 \%-55 \%$ ), percent who arrive by ambulance (range 20\%-23\%), frequency of presenting chief complaints, and arrival times. $^{2}$

All 235,928 visits to the four EDs (range 56,345-62,590 visits per ED) during a 1-year period (October 1, 2006, to September 30, 2007, for three sites and October 1, 2005, to September 30, 2006, for one site) were eligible for inclusion. We excluded 5,986 registration errors, 2,537 visits transferred elsewhere and not treated in the study EDs, 871 visits with multiple missing time or invalid discharge times, 7,775 visits from patients who left without being seen, 2,313 visits from patients who left against medical advice, and 407 ED deaths. This resulted in a final sample size of 216,039 ED visits across the four sites (range 48,896-58,316 visits per ED).

\section{Data Collection and Processing}

From the clinical information system at each ED, we extracted the following electronic information: 1) date and time of registration; 2) date and time of bed placement; 3) date and time of initial contact with physician or midlevel provider (if available); 4) date and time of disposition decision as measured by electronic bed request; 5) date and time of ED discharge or transfer to an inpatient ward if admitted; 6) disposition status; 7) demographic characteristics (age, sex, and insurance status); 8) acuity level; 9) mode of arrival; and 10) chief complaint. Each of the study EDs has a different electronic clinical information system. However, the data are documented in a similar way, which facilitated analysis across the four sites.

Based on the service data above, we divided ED LOS into three phases: 1) waiting room time, defined as time from ED registration to ED bed placement; 2) treatment time, defined as time from ED bed placement until ED providers' disposition decision for admitted patients, and ED bed placement to ED discharge for discharged patients; and 3) boarding time, defined as time from ED providers' disposition decision to ED-hospital transfer for admitted patients. If ED bed placement time was missing, we used time to initial contact with an EP or midlevel provider. If patients who arrived by ambulance were taken directly to a treatment room, their waiting room time was zero and treatment time began at the time of bed placement. Seven percent of the ED visits were missing a data element needed to calculate the waiting room time or boarding time (i.e., either bed placement time or disposition decision for admitted patients).

All of the sites use the ESI to triage patients. The ESI is a five-level triage system that prioritizes patients based on their severity of illness and number of ED resources anticipated. ${ }^{3,4}$ A score of 1 signifies patients of the highest acuity who require immediate, acute care resources, and a score of 5 is given to the lowest acuity patients who do not require urgent services.

Each study ED documents the patient's reason for the ED visit using a standard list of chief complaints with the option of free text. To standardize the chief complaints across the four sites, we classified each chief complaint into one of 10 groups based on the Reason for Visit Classification System used by the National Hospital Ambulatory Medical Care Survey: 1) injury; 2) digestive; 3) nervous system, eyes, or ears; 4) cardiovascular; 5) psychiatric; 6) respiratory; 7) genitourinary; 8) skin; 9) musculoskeletal; and 10) general symptoms. ${ }^{5}$

Finally, we also used temporal factors (arrival day of week and arrival hour) and crowding measures to predict the distribution of ED LOS. In the regression models, we used indicator variables to represent each day of the week. To make the models more parsimonious, we represented the diurnal pattern of ED LOS with six sine and cosine parameters that were fitted from three cosine curves rather than using 24 indicator variables for each hour of the day. ${ }^{6}$ We included the ED occupancy rate and the inpatient medicine bed occupancy rate at the time of each patient's arrival as our crowding measures. The ED occupancy rate was calculated as the ratio of the total number of patients in the ED in a given hour divided by the total number of treatment spaces (excluding hallway locations) staffed during that hour. The hourly inpatient medicine bed occupancy rate was calculated as the ratio of the total number of inpatients occupying a bed assigned to one of the divisions of the department of medicine in a given hour divided by the total number of department of medicine beds. For three of the sites, the inpatient medicine bed occupancy rate was measured hourly during the entire study period, and at one site it was measured every 6 hours. At this site, we linearly interpolated the rate between each measurement period. ${ }^{2}$

\section{Data Analysis}

All analyses were conducted separately for each of the four sites. First, we used multiple imputation methodology to impute missing service completion times for $7 \%$ of the patients missing either bed placement time or admission decision time. Using patients with complete data, we predicted the fraction of waiting room time that was part of the waiting room time and treatment time for patients who had both using linear regression for an empirical logit transformation of the observed fraction. The key predictors in this model included patient (age, sex, insurance status, mode of arrival), clinical (chief complaint and acuity level), temporal (time of day and day of week), and crowding factors (ED occupancy rate and inpatient medicine bed rate). A similar model was estimated for the fraction of boarding time that was part of treatment time and 
boarding time. Five imputed data sets were generated using these prediction equations. These multiple data sets were used to make valid inferences about the predictors of the service completion times. ${ }^{7,8}$ All results presented in this paper include the imputed data.

Second, we estimated the probability densities of waiting room time, treatment time, and boarding time using kernal density estimation. ${ }^{9}$ We displayed the distribution of the service completion time on a log-transformed scale. All remaining analyses were performed using the original time values (not log transformed). Third, we compared the median service completion times by patient, clinical, temporal, and crowding characteristics. Fourth, we conducted stratified analyses to explore for possible interaction effects between the patient, clinical, temporal, and crowding factors on the different service completion outcomes.

Fifth, we used multivariate QR to estimate the 10th, 50th, and 90th percentiles of the waiting room time, treatment time, and boarding time distributions as a function of the patient, clinical, temporal, and crowding factors. All main effects were included in the final models regardless of statistical significance, whereas interaction terms were only retained if the p-value for the regression coefficient was $<0.05$. For each of the final QR models, we provide an estimate of the intercept, which is the estimated service completion time when all of the predictor variables in the model are equal to the reference level (see Results and tables for specification of reference levels). The regression coefficients for each predictor can be interpreted as the change in minutes in service completion time in a given quantile of the conditional distribution by a unit change (i.e., 10-year increase in age, male vs. female, acuity level 2 vs. 3, etc.) in the predictor variable. The total service completion time associated with a specific predictor when it is not at the reference level can be estimated by adding the intercept estimate and the regression coefficient estimate for that variable together. The influence of the temporal factors on the outcomes are displayed by figures, because the regression coefficients associated with the sine and cosine functions (time of day) are not directly interpretable.

We were unable to model service completion times of acuity level 1 patients at sites $B$ and D because the sample sizes were too small (367 and 38, respectively). At sites $\mathrm{A}$ and $\mathrm{C}$, we were able to fit models that only included main effects. There was not sufficient sample size $(2,699$ and 1,421 , respectively) to include all of the interaction terms, so we modeled acuity level 1 patients separately from acuity level 2-5 patients. Thus, the majority of the results presented in this article focus mainly on the results for the acuity level $2-5$ patients at all of the sites.

Finally, to evaluate the predictive accuracy of the QR models, we plotted the observed and predicted 10th, 50th, and 90th percentile service completion times by day of week and time of day and compared how closely they matched each other. We also estimated the proportion of patients who fell below the predicted 10th, 50th, and 90th percentiles for different categories of the predictor variables (acuity levels, mode of arrival, etc.) and compared these to the nomi- nal $0.1,0.5$, and 0.9 values, respectively. In this comparison, we used a 10-fold cross-validation so that the same data were not used to estimate and check the models. ${ }^{10}$ All analyses were conducted in SAS version 9.1 (SAS Institute, Cary, NC) and $\mathrm{R}$ version 2.7.0 (http://www.r-project.org).

\section{RESULTS}

Figure 1 displays the probability distributions of waiting room time, treatment time, and boarding time on a logarithmic scale. Waiting room time remains highly positively skewed even on a logarithmic scale. The median waiting room time ranged from 15 to 44 minutes across the four sites; the 90th percentile ranged from 105 to 222 minutes. The 90th percentile treatment times ranged from 6.6 hours at site D to 10.5 hours at site B. At the 90th percentile, patients at site $\mathrm{C}$ waited two to three times longer for an inpatient bed (20.5 hours) compared to patients at the other sites (range 6.4-8.8 hours; data not shown).

Tables 2-5 provide the site-specific QR estimates of the effect of different factors on the 10th, 50th, and 90th percentiles of the three service completion times. The intercept values in Tables 2-5 are estimates from the QR models of the waiting room time, treatment time, and boarding time for a 40-year-old male, covered by commercial insurance, with an acuity level 3 general symptom chief complaint, who arrives without ambulance transport, at midnight on Saturday when the ED occupancy rate is at $100 \%$ and the inpatient medicine bed occupancy rate is $85 \%$. In all three of these tables, the intercept values show that the difference in time between the 50th and 90th percentiles is much greater than the difference in the 10th and 50th percentiles.

For waiting room time, the most influential patient characteristic was mode of arrival. At all of the study sites, patients who arrived by ambulance waited less time to be placed in a room (50th percentile range -18 to -54 across the sites) compared to patients who did not arrive by ambulance. However, the effect of mode of arrival was much larger at the 50th and 90th percentiles compared to the 10th percentile. Chief complaint, acuity level, and the interaction of chief complaint and acuity level were all significant predictors of waiting room time. Patients who presented with injuries (50th percentile range -5 to -9 minutes across the sites), cardiovascular complaints (50th percentile range -3 to -11 minutes across the sites), or respiratory complaints (50th percentile range -2 to -8 minutes across the sites) were more quickly placed in a room compared to patients with a general symptom complaint across the four sites. Acuity level exerted the strongest effect on waiting room time, particularly at the 50th and 90th percentiles of the distribution. A $10 \%$ increase in the ED occupancy rate had a much bigger effect on waiting room time across the sites at the 50th (range 7 to 12 minutes) and 90th (range 15 to 33 minutes) percentiles compared to the 10th percentile (range 0 to 2 minutes).

We observed a strong interaction effect between acuity level and temporal factors (i.e., time of day and day of week) on waiting room time at all four sites (see 

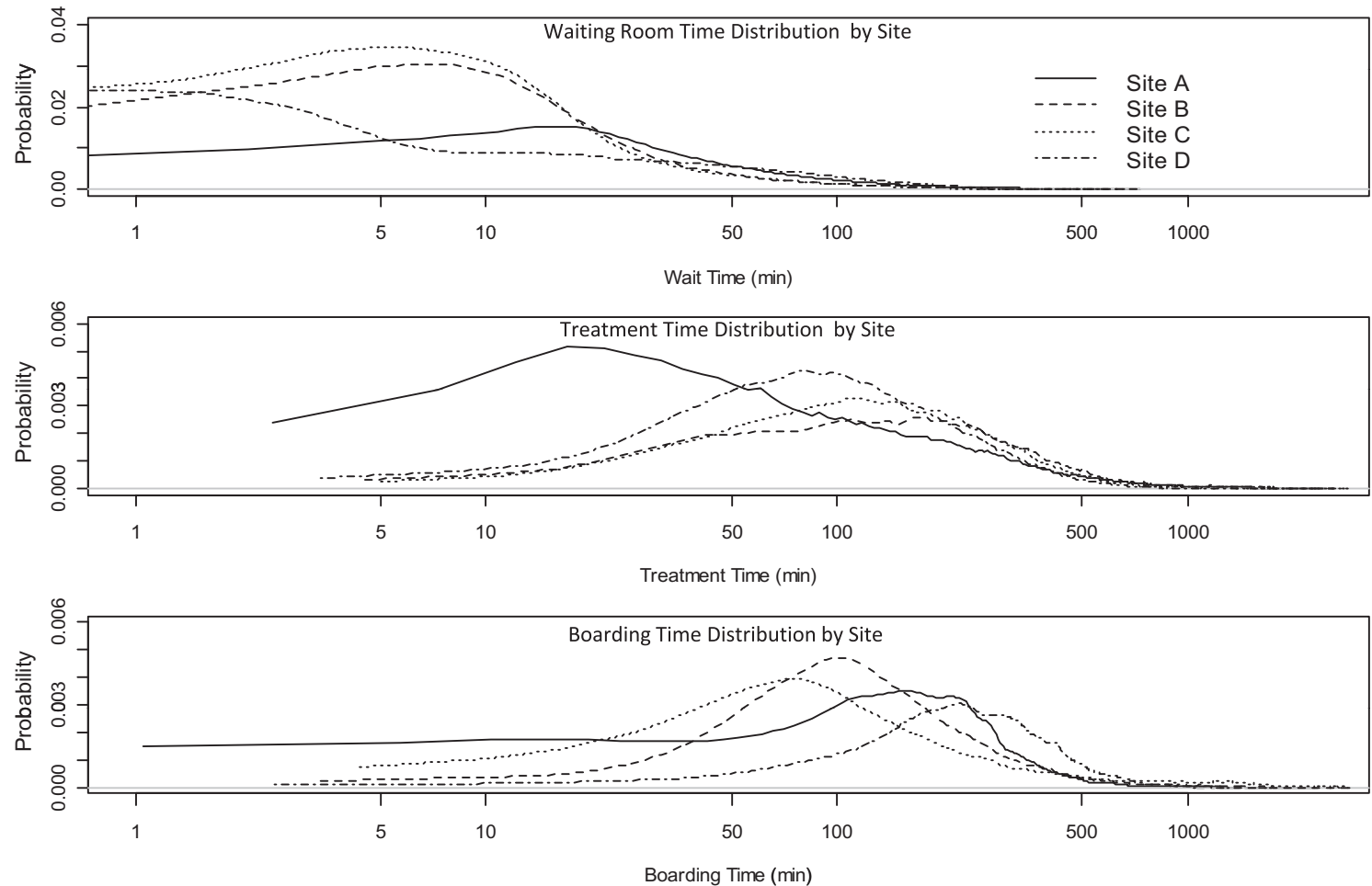

Figure 1. Probability density distribution (smoothed histogram) of waiting room time, treatment time, and boarding time on a log scale by site.

Table 2

Change (Minutes) Associated With Patient, Clinical, and Crowding Factors on the 10th, 50th, and 90th Percentiles of Waiting Room Time*

\begin{tabular}{|c|c|c|c|c|c|c|c|c|c|c|c|c|}
\hline \multirow[b]{2}{*}{ Variables in Model $\dagger$} & \multicolumn{3}{|c|}{$\begin{array}{c}\text { Site A } \\
(n=54,847)\end{array}$} & \multicolumn{3}{|c|}{$\begin{array}{c}\text { Site B } \\
(n=53,980)\end{array}$} & \multicolumn{3}{|c|}{$\begin{array}{c}\text { Site C } \\
(n=48,896)\end{array}$} & \multicolumn{3}{|c|}{$\begin{array}{c}\text { Site D } \\
(n=58,316)\end{array}$} \\
\hline & $10 \%$ & $50 \%$ & $90 \%$ & $10 \%$ & $50 \%$ & $90 \%$ & $10 \%$ & $50 \%$ & $90 \%$ & $10 \%$ & $50 \%$ & $90 \%$ \\
\hline Intercept & 14 & 95 & 304 & 5 & 44 & 153 & 5 & 36 & 121 & 0 & 50 & 119 \\
\hline \multicolumn{13}{|l|}{ Patient } \\
\hline Age (10-year increase) & 0 & - & 5 & 0 & 0 & -1 & 0 & -1 & -1 & - & -1 & - \\
\hline $\begin{array}{l}\text { Female (vs. male) } \\
\text { Insurance (vs. commercial) }\end{array}$ & 1 & - & \multicolumn{2}{|c|}{ Insurance (vs. commercial) } & 4 & & 1 & 1 & 4 & - & 4 & 7 \\
\hline Medicaid & - & 7 & - & 1 & 3 & - & 1 & 2 & 6 & - & 9 & 11 \\
\hline Medicare & 2 & 11 & 33 & - & - & - & - & 3 & - & - & 8 & 13 \\
\hline Self-pay & 1 & 8 & 24 & 2 & 4 & - & 2 & - & - & - & 3 & - \\
\hline Ambulance arrival (vs. not ambulance) & -5 & -26 & -41 & -6 & -29 & -33 & -6 & -18 & -20 & - & -54 & -71 \\
\hline \multicolumn{13}{|l|}{ Clinical } \\
\hline \multicolumn{13}{|l|}{ Chief complaint (vs. general) } \\
\hline Injury & -2 & -9 & -18 & - & -8 & -14 & - & -5 & -17 & - & -9 & -17 \\
\hline Digestive & - & 9 & - & 2 & 6 & 16 & 2 & 2 & - & - & 3 & 9 \\
\hline Cardiovascular & - & - & - & - & -3 & - & - & -5 & -6 & - & -11 & -13 \\
\hline Psychiatric & - & -11 & 48 & 2 & -9 & -18 & 2 & - & -9 & - & -8 & - \\
\hline Nervous/eye/ears & - & - & - & 2 & - & - & 2 & -2 & -12 & - & -7 & -9 \\
\hline Respiratory & - & - & -15 & - & -5 & - & - & -2 & -4 & - & -8 & -13 \\
\hline Genitourinary & - & 6 & - & 2 & 3 & - & 2 & - & 10 & - & - & - \\
\hline Skin & - & - & - & - & - & - & - & - & - & - & - & - \\
\hline Musculoskeletal & - & - & 15 & 3 & 3 & - & 3 & - & -7 & - & - & - \\
\hline $\begin{array}{l}\text { Acuity (vs. level 3) } \$ \\
\text { Level } 2\end{array}$ & & & & & & & & & & & & \\
\hline Level 2 & -9 & -70 & -192 & -4 & -33 & -118 & 0 & -15 & -38 & - & -33 & -33 \\
\hline Level 4 or 5 & -1 & -40 & -101 & - & -17 & -58 & 3 & -6 & -36 & - & -4 & -7 \\
\hline \multicolumn{13}{|l|}{ Crowding } \\
\hline ED occupancy ( $10 \%$ increase) & 2 & 12 & 33 & 0 & 9 & 27 & 0 & 7 & 21 & - & 8 & 15 \\
\hline Medicine bed ( $10 \%$ increase) & 0 & 3 & 8 & 0 & -2 & -10 & 0 & -2 & -14 & - & - & - \\
\hline
\end{tabular}

*Nonsignificant estimates $(p>0.05)$ are shown by a dash $(-)$.

†Other covariates in each regression model included time of day, day of week and interaction of chief complaint $\times$ acuity, time of day $\times$ day of week $\times$ acuity, and time of day $\times$ day of week $\times$ mode of arrival (only in site $C$ ).

$\ddagger$ Acuity level 1 patients not included in this model. 
Table 3

Change (Minutes) Associated With Patient, Clinical, and Crowding Factors on the 10th, 50th, and 90th Percentiles of Treatment Time*

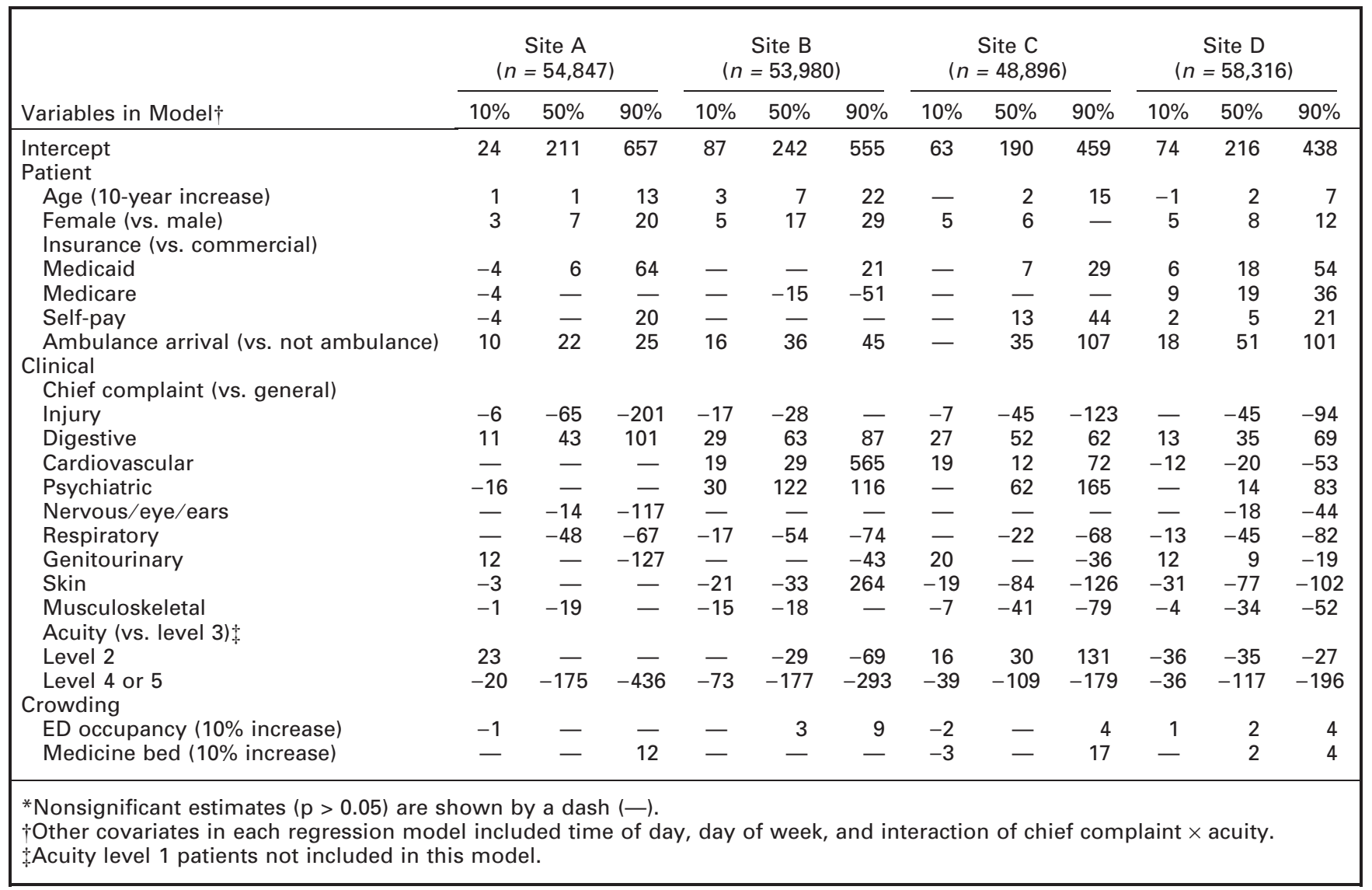

Figure 2). The largest difference in waiting room time by acuity level was observed at approximately 6:00 PM. There is little temporal variation in median waiting room time for acuity level 1 patients. However, the waiting room time of acuity level 2 patients varied significantly by day of week and time of day at all sites except site B. Acuity level 3 patients waited the longest across the four sites, and their waiting room times were most influenced by temporal factors compared to other acuity level patients. The waiting room times at sites $\mathrm{B}$ and $\mathrm{C}$ were much shorter on the weekends compared to the weekdays and varied much less by acuity compared to during weekdays.

Table 3 illustrates the strong effect that the clinical factors had on treatment time compared to patient or crowding factors. In general, patients who presented with an acuity level 3 general symptom complaint waited longer than most others, except for patients with digestive complaints (50th percentile range 35 to 63 minutes) or psychiatric complaints (50th percentile range 14 to 122 minutes). Across all of the sites, acuity level 4 and 5 patients (50th percentile range -109 to 177 minutes) were treated faster than acuity level 3 patients. There was also a strong interaction effect between chief complaint and acuity level (see Table 4). At all study sites, skin, injury, and respiratory problems were associated with the shortest median treatment times for acuity level 2 and 3 patients. Patients who presented with psychiatric complaints such as suicide ideation, depression, or alcohol abuse had the longest treatment times, regardless of acuity level. For acuity level 2-5 patients, the 90th percentile treatment times were two to five times greater than the 50th percentile treatment times depending on the specific chief complaint, acuity level, and site.

Patient, clinical, and crowding factors had a modest influence on boarding time (see Table 5). The most consistent effect on boarding time at all of the sites and percentile distributions were the crowding factors. A $10 \%$ increase in the inpatient medicine bed occupancy rate (at the 50th percentile range is 13 to 58 minutes) resulted in a longer boarding time compared to the same increase in the ED occupancy rate (at the 50th percentile range is 3 to 14 minutes). Both crowding factors had larger effects on the right tail of the boarding time distribution.

Figure 3 displays the observed and predicted 10th, 50th, and 90th percentile waiting room times, treatment times, and boarding times at all four sites. The waiting room time at the 90th percentile ranged between 15 minutes and 4 hours depending on the time of day, day of week, and site. In addition to the strong influence of temporal factors on waiting room time, Figure 3 also shows that time of day and day of week influence treatment time and boarding time (to a lesser degree than waiting room time). At site C, the 90th 
Table 4

Adjusted 10th, 50th, and 90th Percentiles of Treatment Time by Acuity Level and Chief Complaint*

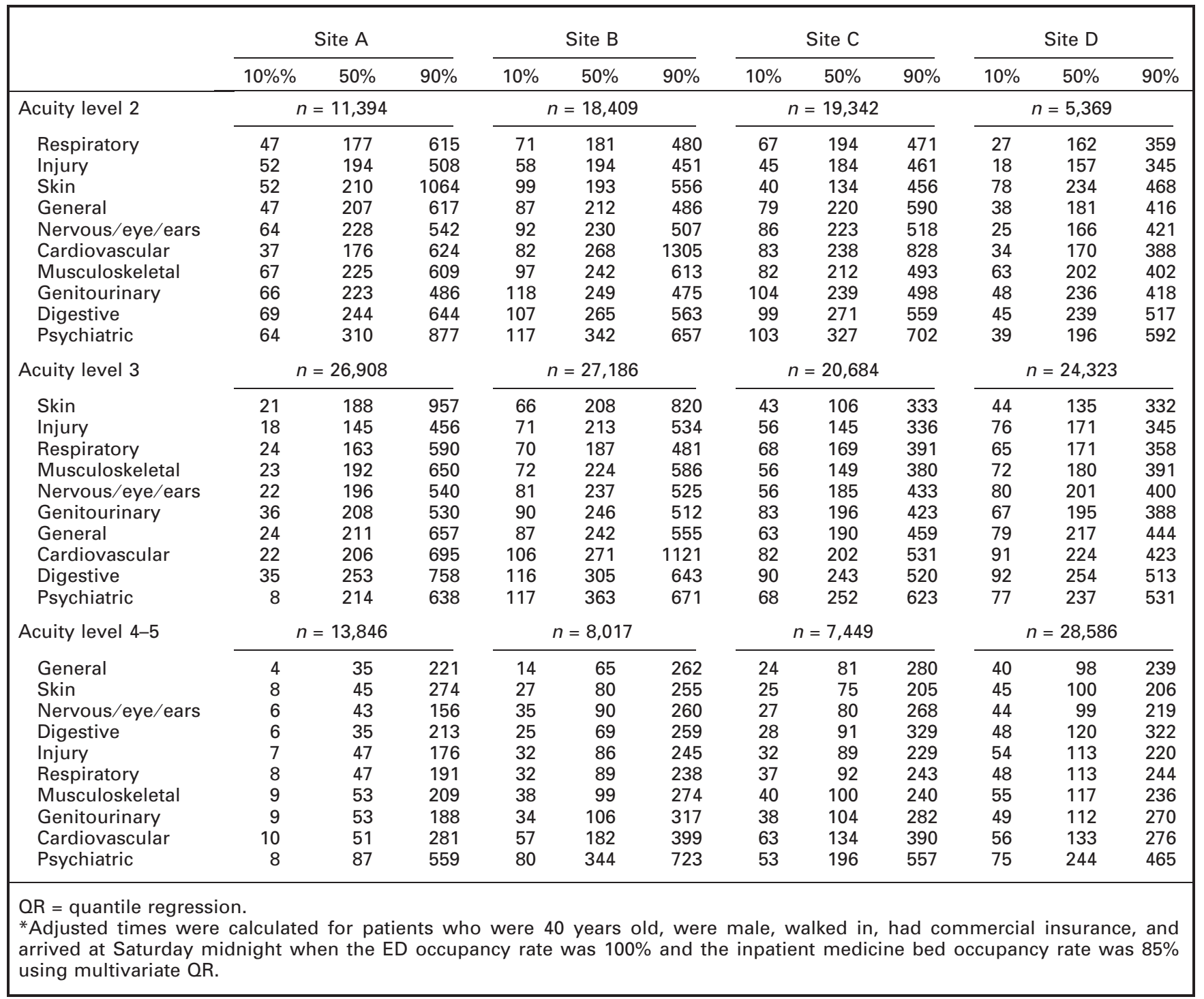

percentile boarding times were much longer during the week compared to weekends.

Figure 3 also demonstrates close agreement between the observed and predicted 10th, 50th, and 90th percentile waiting room time, treatment time, and boarding time distributions. Further evidence of the accuracy of the models is displayed in Table 6 by the proportion of predicted estimates that fell within the 10th, 50th, and 90th percentile distributions for the different phases of emergency care. At each site and acuity level, the estimates from the QR models fell within $1 \%$ to $2 \%$ of the observed 10th, 50th, and 90th percentile distributions.

\section{DISCUSSION}

It is well recognized that perceptions of a service organization are significantly affected by how well it performs not only for typical customers but also for those at the extremes. This study demonstrates that $\mathrm{QR}$ is a useful method for characterizing the typical LOS expe- rience, as well as at both ends of the LOS distribution. At all of the study EDs, we observed large variability in ED service completion times. Waiting room time was largely determined by day of week, time of day, and acuity level. In contrast, the strongest predictors of treatment time were acuity level and chief complaint. Temporal factors also influenced boarding time. With the exception of mode of arrival, patient characteristics had minimal influence on ED service completion times. The influence of some of the factors varied significantly across the percentile distributions. Our data suggest that EDs across the country could use QR to more fully understand how the efficiency of ED care varies and to identify factors whose effects may not be constant across the service completion time distributions.

Quantile regression is appropriate when the predictor variables may affect the outcome differently at different parts of the distribution. The ED service completion distributions at all of the study sites were highly skewed, with long right tails. The difference in the time between the 10th percentile and the 50th 
Table 5

Change (Minutes) Associated With Patient, Clinical, and Crowding Factors on the 10th, 50th, and 90th Percentiles of Boarding Time*

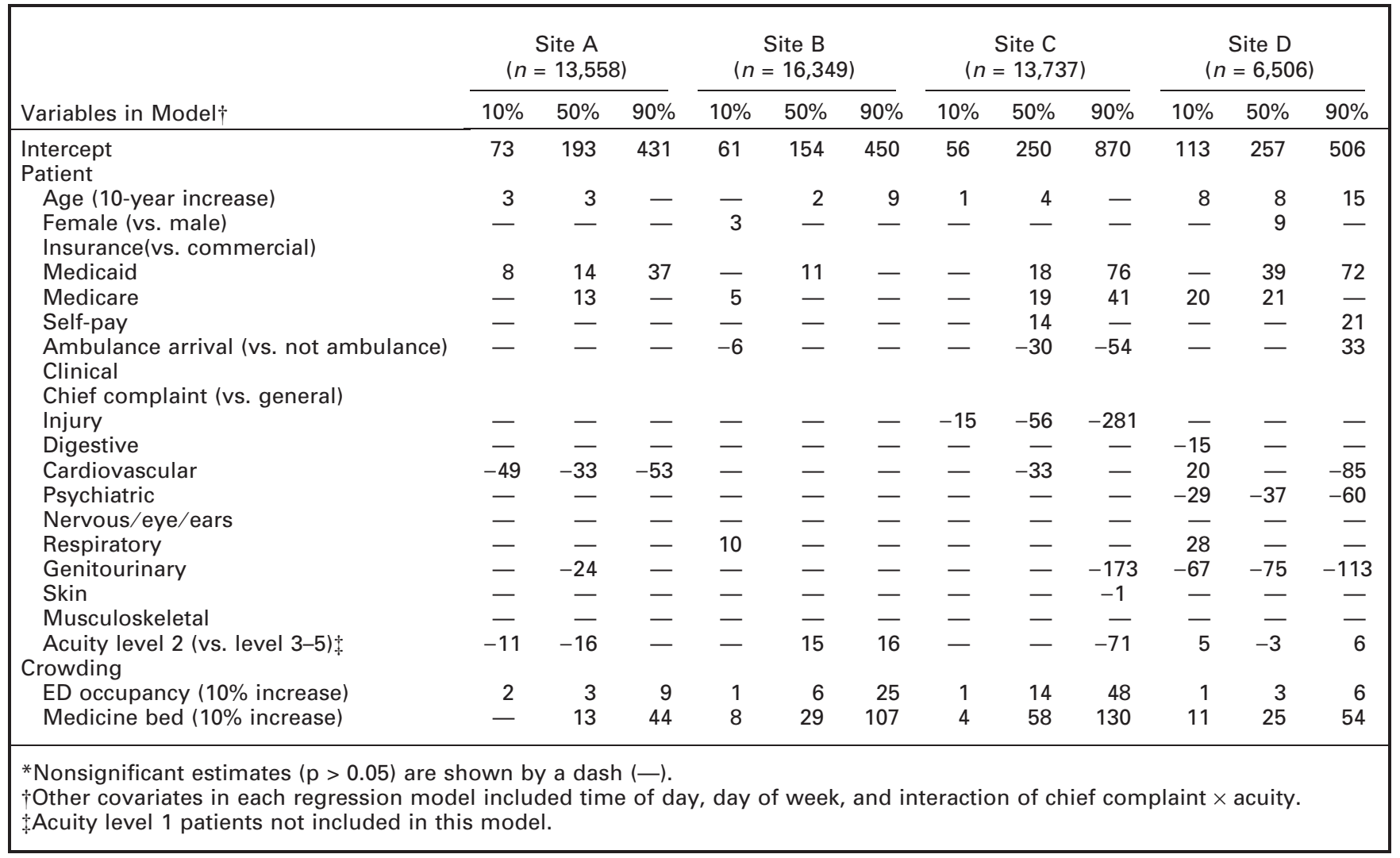
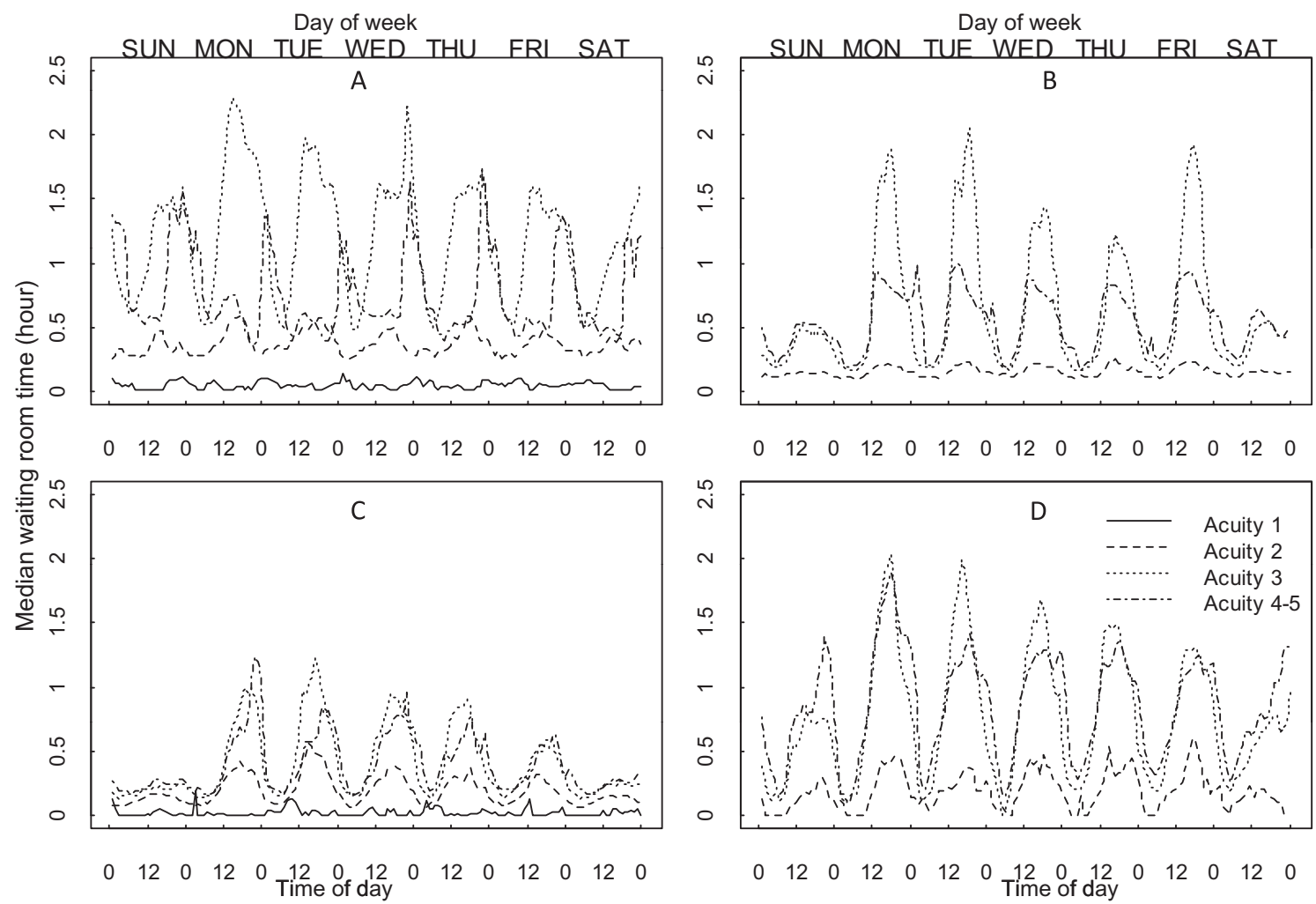

$\begin{array}{lllllll}0 & 12 & 0 & 12 & 0 & 12 & 0 \\ \text { Time of day } & 0 & 12 & 0 & 12 & 0\end{array}$

Figure 2. Observed median waiting room time (in hours) by acuity and site. 
A

Day of week
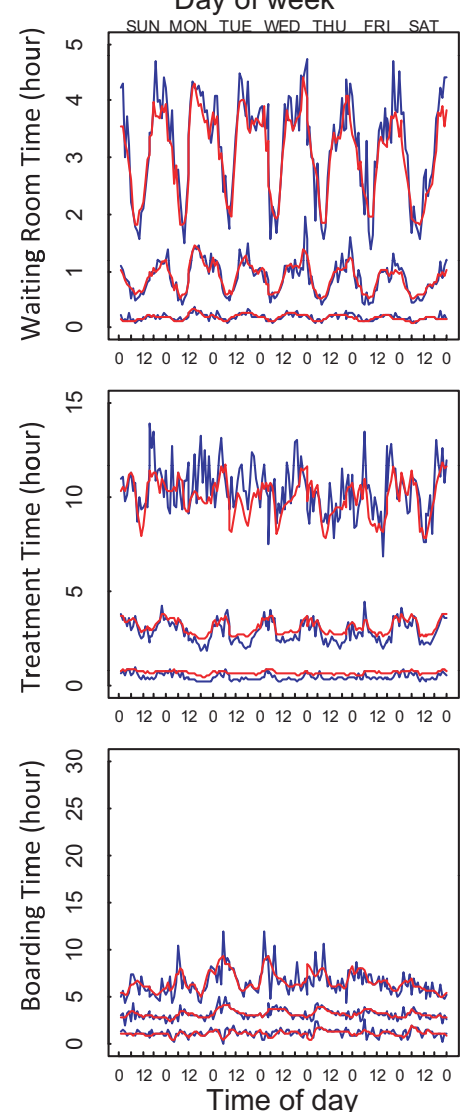

B

Day of week
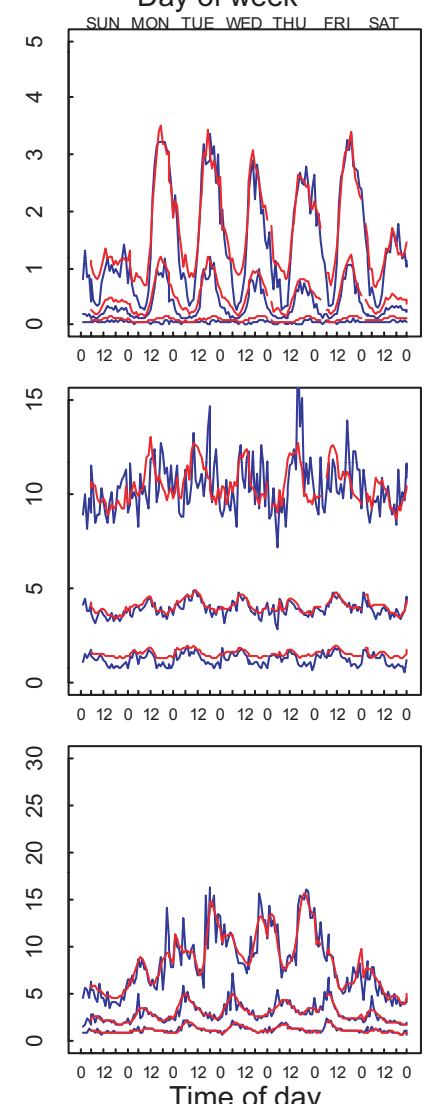

C

Day of week
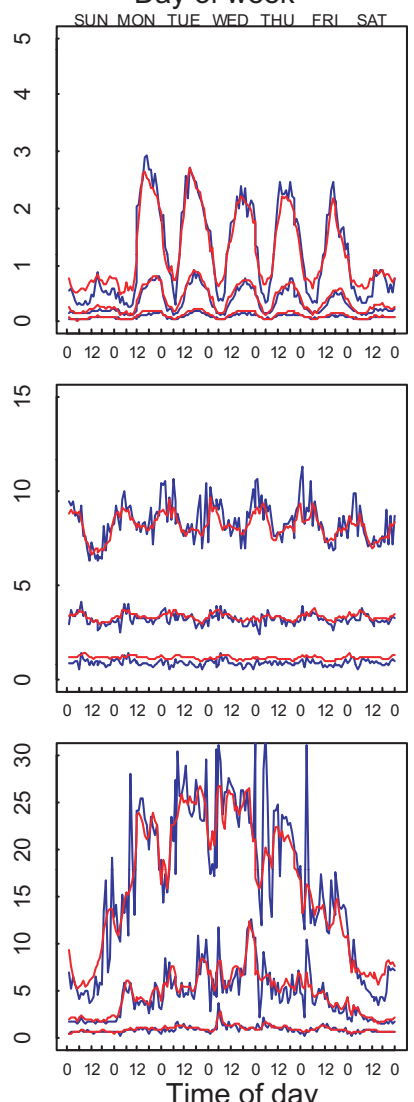

D

Day of week
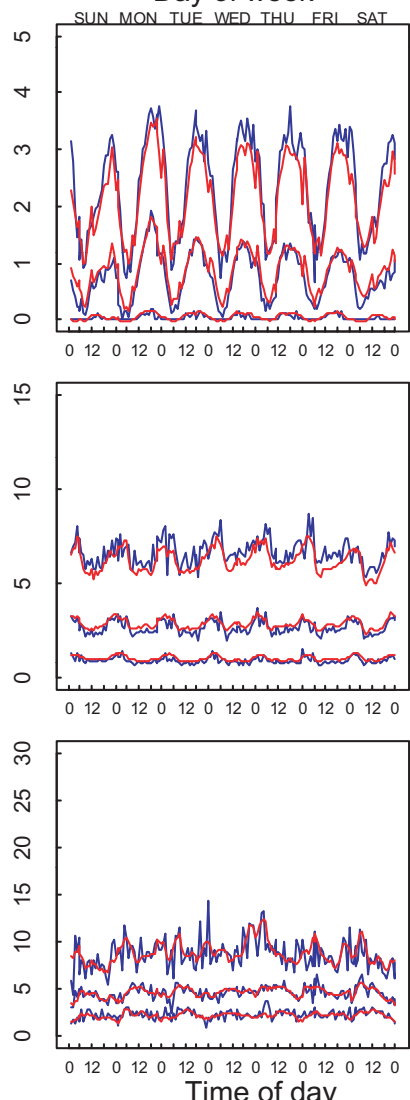

Figure 3. Observed (blue lines) and predicted (red lines) 10th, 50th, and 90th percentiles (in hours) of waiting room time, treatment time, and boarding time by day of week, hour of day, and site.

\begin{tabular}{|c|c|c|c|c|c|c|c|c|c|c|c|c|c|}
\hline \multirow[b]{2}{*}{ ED LOS } & \multirow{2}{*}{$\begin{array}{l}\text { Acuity } \\
\text { Level }\end{array}$} & \multicolumn{3}{|c|}{ Site A } & \multicolumn{3}{|c|}{ Site B } & \multicolumn{3}{|c|}{ Site C } & \multicolumn{3}{|c|}{ Site D } \\
\hline & & 10th & 50th & 90th & 10th & 50th & 90th & 10th & 50th & 90th & 10th & 50th & 90th \\
\hline & & \multicolumn{3}{|c|}{$n=54,847$} & \multicolumn{3}{|c|}{$n=53,980$} & \multicolumn{3}{|c|}{$n=48,896$} & \multicolumn{3}{|c|}{$n=58,316$} \\
\hline $\begin{array}{l}\text { Waiting } \\
\text { room time }\end{array}$ & $\begin{array}{c}1 \\
2 \\
3 \\
4-5\end{array}$ & $\begin{array}{r}0.098 \\
0.098 \\
0.103 \\
0.099 \\
n\end{array}$ & $\begin{array}{l}0.497 \\
0.504 \\
0.505 \\
0.495 \\
54,847\end{array}$ & $\begin{array}{l}0.900 \\
0.904 \\
0.896 \\
0.899\end{array}$ & $\begin{array}{r}-\overline{0} \\
0.098 \\
0.104 \\
0.096\end{array}$ & $\begin{array}{r} \\
-\overline{4} \\
0.596 \\
0.500 \\
=53,98\end{array}$ & $\begin{array}{c}-\overline{0} \\
0.902 \\
0.895 \\
0.899\end{array}$ & $\begin{array}{l}0.103 \\
0.103 \\
0.095 \\
0.096\end{array}$ & $\begin{array}{r}0.502 \\
0.498 \\
0.500 \\
0.499 \\
=48,89 \\
\end{array}$ & $\begin{array}{l}0.899 \\
0.904 \\
0.896 \\
0.896\end{array}$ & $\begin{array}{c}- \\
0.097 \\
0.102 \\
0.101\end{array}$ & $\begin{array}{r}-\overline{498} \\
0.500 \\
0.500 \\
=58,31\end{array}$ & $\begin{array}{c}-\overline{898} \\
0.902 \\
0.899\end{array}$ \\
\hline $\begin{array}{l}\text { Treatment } \\
\text { time }\end{array}$ & $\begin{array}{c}1 \\
2 \\
3 \\
4-5\end{array}$ & $\begin{array}{r}0.102 \\
0.104 \\
0.098 \\
0.099\end{array}$ & $\begin{array}{l}0.499 \\
0.495 \\
0.499 \\
0.502 \\
13,558\end{array}$ & $\begin{array}{l}0.897 \\
0.903 \\
0.899 \\
0.901\end{array}$ & $\begin{array}{l}-\overline{100} \\
0.103 \\
0.099\end{array}$ & $\begin{array}{r} \\
0 . \overline{497} \\
0.503 \\
0.501 \\
=16,349\end{array}$ & $\begin{array}{c}-\overline{0} \\
0.904 \\
0.896 \\
0.895\end{array}$ & $\begin{array}{l}0.104 \\
0.104 \\
0.095 \\
0.101\end{array}$ & $\begin{array}{r}0.498 \\
0.495 \\
0.499 \\
0.506 \\
=13,73\end{array}$ & $\begin{array}{l}0.904 \\
0.904 \\
0.905 \\
0.896\end{array}$ & $\begin{array}{c}-\overline{103} \\
0.098 \\
0.103\end{array}$ & $\begin{array}{r}-\overline{504} \\
0.496 \\
0.495 \\
=6,506\end{array}$ & $\begin{array}{c}-\overline{895} \\
0.901 \\
0.905\end{array}$ \\
\hline
\end{tabular}

percentile was much smaller than the difference between the 50th and 90th percentile. Many patients are waiting much longer than the average or the median. High performing service industries set their delivery targets at the 90th percentile or higher, not at the median. ${ }^{11}$ In the ED setting, we need to focus not only on the middle of the distribution, but also on extreme events, especially for patients with long 
waiting room and/or boarding times, because these phases represent little value to patients.

The influence of some factors on ED service completion time varied substantially across the distribution. For example, at site $\mathrm{A}$, at the median of the distribution of waiting room time, patients with psychiatric complaints waited 11 minutes less compared to patients with general symptom complaints. However, at the 90th percentile, psychiatric patients waited 48 minutes longer than patients with general symptom complaints. This site needs to determine why the disparity does not exist at the center of the distribution, but does exist at the right tail. Similarly, at site C, the boarding times of patients with injuries are significantly less at the 90th percentile compared to the 10th percentile. Site C could investigate why injury is more influential at the 90th percentile compared to the 10th.

In this study, temporal factors (i.e., time of day and day of week) were important predictors of waiting room time and boarding time. To a large extent, they are important predictors because they are surrogate measures of the demand for ED and inpatient services and the resources available to meet the demand. Demand for ED services, as measured by the ED arrival rate, is highest in the late morning and afternoon hours and lowest at night and in the early morning hours. ${ }^{12,13}$ The ED arrival rate also varies by day of week. Depending on staffing and ED capacity, ED waiting room times will closely mirror the ED arrival rate pattern with a modest lag unless there is a boarding problem, which will also independently influence the waiting room time distribution. ${ }^{2}$ Likewise, boarding times also vary by time of day and day of week because the demand for inpatient beds by ED patients and patients from other venues peaks at predictable times of the day and days of the week. The crowding factors that we included in the models had a modest effect on waiting room time and boarding time after controlling for time of day and day of week. This was expected given the colinearity between the crowding measures and the temporal factors.

We also observed that the effect of temporal factors on ED waiting room time varied significantly by acuity level. This interaction effect is not surprising given that EDs prioritize and organize the delivery of emergency care according to triage level. For acuity level 1 patients who presented with life-threatening conditions, there was no significant variation in ED waiting room time by time of day or day of week at the study sites. For acuity level 2 and 3 patients, who are considered to have emergent and urgent problems, respectively, the variation in waiting room time by temporal factors varied by order of magnitude (temporal variation was much greater for acuity level 3 vs. 2 patients). The nonurgent patients (i.e., acuity levels 4 and 5) had shorter wait times than acuity level 3 patients, because patients who presented with nonurgent problems were seen in the "urgent care" or "fast track" areas of the study EDs. Acuity level 3 patients waited the longest at all four study sites because they were assigned the lowest level of priority among the patients treated in the main area of the ED.

Clinical factors, that is, acuity and chief complaint, were also significant predictors of ED LOS, particularly
ED treatment time. Asaro and colleagues ${ }^{1}$ also found that acuity level and chief complaint were the most important determinants of ED and fast track treatment times. To the best of our knowledge, this is the first study to identify a significant interaction effect between acuity level and chief complaint on ED service completion times. For ED treatment time, the variation in service completion time by acuity level for a specific chief complaint is most likely due to the differences in testing and treatment required depending on the urgency and complexity of the condition.

There was considerable variation in service completion times across the sites. Some of the variation is likely due to organizational differences among the sites. For example, site $\mathrm{C}$ has the shortest waiting room time of all the sites, most likely because it has the largest treatment capacity ( 9 to 15 more treatment spaces in the main ED than the other EDs). The 90th percentile treatment times at sites $\mathrm{A}$ and $\mathrm{B}$ were considerably longer than the other two sites; however, these two sites have observation units, and the care patients received in the ED observation units was included in the treatment times reported in this analysis. The reasons for other variations in service completion times are less clear. For example, each of the study EDs has a fast track, yet there was large variation in the waiting room time and treatment time of patients who presented with nonurgent problems (i.e., acuity level 4 and 5 ) across the sites. Examining differences in outcome by site and identifying best practices is a fruitful area of future research.

\section{LIMITATIONS}

The results of this study must be considered in the context of the following limitations. First, we used separate models to estimate the different phases of emergency care. We did not attempt to predict total ED LOS. Predicting total ED LOS is more challenging because it requires accurately predicting the probability of admission and modifying the service completion estimates by the probability of admission. Second, these models provide estimates of service completion times at the time of triage. We have not yet incorporated into the models the effect of ordering different tests or specific test results, services requested, or procedures rendered on estimates of the service completion times. ${ }^{1,14}$ As care is delivered to ED patients, service completion estimates could be updated based on the effect of different evaluation and treatment activities and provided to patients during the course of their ED visit.

A third limitation is that the sample size was too small to develop comprehensive models for acuity level 1 patients; however, they are the least likely to need service completion estimates because their care is typically expedited by the severity of their illness or injury. Fourth, we only had a single year of data from each site, limiting our ability to estimate the effect of seasonality on the ED service completion times. However, this could easily be estimated by using ED visit data from multiple years. In addition, with more data, we could have made more clinically distinct groups out of the 10 general chief complaint categories and estimated the 
service completion times with more precision. Fifth, we did not verify the accuracy of the electronic data, but some of the data are time stamped automatically, such as registration time and decision time to admit. Further, all of the study sites depend on these data to monitor operations, so they are being reviewed and checked regularly.

Finally, all of the sites are academic EDs; there were no community hospital EDs included in the study. None of the EDs consistently met industry standards for all phases of emergency care. Regardless of how fast or slow a particular phase of care was completed at the four different EDs, QR proved to be a robust method for predicting ED service completion times. The results suggest that the overall approach should generalize well to other EDs, and the models can be customized to reflect any unique influences that different factors have on service delivery at a specific ED.

\section{CONCLUSIONS}

Quantile regression proved to be a useful method for characterizing the service completion experience of not only typical ED patients, but also of patients who waited much shorter or longer times. In this study, quantile regression illustrated how highly variable the length of stay experience is among ED patients at four academic centers. Building accurate models of ED service completion times is a critical first step needed to identify barriers to patient flow, begin the process of reengineering the system to reduce variability, and improve the timeliness of care provided.

\section{References}

1. Asaro PV, Lewis LM, Boxerman SB. The impact of input and output factors on emergency department throughput. Acad Emerg Med. 2007; 14:235-42.

2. McCarthy ML, Zeger SL, Ding $R$, et al. Crowding delays treatment and lengthens emergency depart- ment length of stay, even among high acuity patients. Ann Emerg Med. 2009; 54:492-503.

3. Wuerz RC, Milne LW, Eitel DR, Travers D, Gilboy N. Reliability and validity of a new five-level triage instrument. Acad Emerg Med. 2000; 7:236-42.

4. Eitel DR, Travers DA, Rosenau AM, Gilboy N, Wuerz RC. The Emergency Severity Index triage algorithm version 2 is reliable and valid. Acad Emerg Med. 2003; 10:1070-80.

5. Schneider D, Appleton L, McLemore T. A reason for visit classification for ambulatory care. Vital Health Stat 2. 1979; 78:1-63.

6. Bloomfield P. Fourier Analysis of Time Series: An Introduction. 2nd ed. New York, NY: John Wiley \& Sons, 2000.

7. Little RJ, Rubin DB. Statistical Analysis With Missing Data. 2nd ed. Hoboken, NJ: John Wiley \& Sons, 2002.

8. Newgard CD, Haukoos JS. Advanced statistics: missing data in clinical research-part 2: multiple imputation. Acad Emerg Med. 2007; 14:669-78.

9. Everitt BS, Hothorn T. A Handbook of Statistical Analyses Using R. 1st ed. Boca Raton, FL: Chapman \& Hall/CRC, 2006.

10. Harrell FE. Regression Modeling Strategies With Applications to Linear Models, Logistic Regression, and Survival Analysis. 1st ed. New York, NY: Springer, 2001.

11. Stevenson WJ. Operations Management. 10th ed. New York, NY: McGraw-Hill/Irwin, 2008.

12. McCarthy ML, Zeger SL, Ding R, Aronsky D, Hoot NR, Kelen GD. The challenge of predicting demand for emergency department services. Acad Emerg Med. 2008; 15:337-46.

13. McCaig LF, Ly N. National Hospital Ambulatory Medical Care Survey: 2000 emergency department summary. Adv Data. 2002; 326:1-32.

14. Gardner RL, Sarkar U, Maselli JH, et al. Factors associated with longer ED lengths of stay. Am J Emerg Med. 2007; 25:643-50.

\section{Dynamic Emergency Medicine is Now Online-only}

Effective with the January 2010 issue, Academic Emergency Medicine is now running Dynamic Emergency Medicine online only. Several accepted submissions that are currently at the publisher will be run in print as before, but all future accepted DynEM papers will run online only. 